\title{
FIELD PREVALENCE OF WOLBACHIA IN THE MOSQUITO VECTOR AEDES ALBOPICTUS
}

\author{
PATTAMAPORN KITTAYAPONG, VISUT BAIMAI, AND SCOTT L. O'NEILL \\ Department of Biology, Faculty of Science, Mahidol University, Bangkok, Thailand; Section of Vector Biology, \\ Department of Epidemiology and Public Health, Yale University School of Medicine, New Haven, Connecticut
}

\begin{abstract}
The endosymbiotic bacteria in the genus Wolbachia have been proposed as a potential candidate to deliver pathogen-blocking genes into natural populations of medically important insects. The successful application of Wolbachia in insect vector control depends on the ability of the agent to successfully invade and maintain itself at high frequency under field conditions. Here, we evaluated the prevalence of Wolbachia infections in a field population of the Wolbachia-superinfected mosquito Aedes albopictus. A field prevalence of $100 \%(n=1,016)$ was found in a single population in eastern Thailand via polymerase chain reaction (PCR) testing of Wolbachia both from individual parent females and their corresponding F1 offspring. This is the first report of accurate Wolbachia prevalence in a field population of an insect disease vector. The prevalence of superinfection was estimated to be $99.41 \%$. All single-infected individual mosquitoes $(n=6)$ were found to harbor group A Wolbachia. For this particular population, none was found to be single-infected with group B Wolbachia. Our results also show that PCR testing of field materials alone without checking F1 offspring overestimated the natural prevalence of single infection. Thus, the confirmation of infection status by means of F1 offspring was critical to the accurate estimates of Wolbachia prevalence under field conditions.
\end{abstract}

\section{INTRODUCTION}

The mosquito Aedes albopictus (Skuse) is native to Asia and the South Pacific and has recently been introduced into the continental United States and South America. ${ }^{1,2}$ This species has been implicated as one of the important vectors of endemic dengue in Southeast Asia. Under experimental conditions, it has been shown to be an efficient vector, ${ }^{3}$ and dengue virus has been recovered from field mosquitoes. ${ }^{4,5}$ For example, Ae. albopictus was reported to be naturally infected with dengue virus during the 1995 outbreak in Mexico. ${ }^{6}$ Similarly, Ae albopictus was observed to participate in viral transmission during dengue hemorrhagic fever outbreaks in Singapore ${ }^{4}$ and on the island of Samui, Thailand. ${ }^{7}$

Wolbachia infection was discovered in Ae. albopictus by Wright and Barr. ${ }^{8}$ They reported the presence of Wolbachia in the ovaries of mosquitoes collected from Thailand. Later, the expression of Wolbachia-mediated cytoplasmic incompatibility in laboratory-bred Ae. albopictus was studied by Kambhampati and others. ${ }^{9,10}$ In 1995, the presence of Wolbachia superinfecions was detected in 12 laboratory populations of Ae. albopictus by restriction fragment length polymorphism of both the $16 \mathrm{~S}$ rRNA polymerase chain reaction (PCR) products when digested with $X b a \mathrm{I}$ and the fts $Z$ PCR products when digested with EcoRV. ${ }^{11}$ Results showed that all colonies except for the Mauritius and Koh Samui strains appeared to be superinfected with strains of A and B group Wolbachia. Individuals from these single-infected colonies exhibited Wolbachia-mediated cytoplasmic incompatibility when crossed with other mainland colonies of the same species. ${ }^{10}$

Wolbachia-induced cytoplasmic incompatibility has been proposed as a potential mechanism to introduce and spread transmission-blocking genes into natural populations of insect vectors in an attempt to modify the vector competence of these populations. ${ }^{12,13}$ The success of this long-term goal for disease control is critically dependent on the ability of Wolbachia to invade a host population and to establish a stable equilibrium prevalence within the target population that is high enough to have a significant impact on disease transmission. ${ }^{14}$ Previous studies have indicated that in Drosophila simulans field populations infected with $w \mathrm{Ri}$ Wolbachia, this stable equilibrium frequency is commonly $\sim 96-97 \% .^{15}$ Although this infection frequency is quite high, it may not be high enough to eliminate disease transmission in an insect vector population, even if all Wolbachia-infected insects were genetically altered so as to be completely unable to transmit pathogens. However, very few reliable data exist in species outside of the genus Drosophila to indicate whether infection frequencies reported in Drosophila are typical for other insect species. A number of studies have reported infection frequencies of different insect species that are based on PCR surveys, but these results are notoriously unreliable because of the common occurrence of false-negative results in the PCR assays used to detect Wolbachia in insects. ${ }^{16}$

In this study, we examined the stable field infection frequency of a natural Wolbachia infection in the vector mosquito, Ae. albopictus, in a region of endemic dengue transmission. In order to circumvent the problems of previous studies in accurately measuring this frequency, we used a combination of PCR detection of Wolbachia in field collected adults as well as in the laboratory-reared F1 progeny of these same individuals.

\section{MATERIALS AND METHODS}

Mosquito specimens. Aedes albopictus mosquitoes were collected weekly for 6 months from August 1999 to January 2000 in their natural habitats in Hua Samrong Subdistrict, Plaeng Yao District, Chachoengsao Province, eastern Thailand. The method of collection was the standard mosquito landing catch. ${ }^{17}$ Live mosquitoes were brought back to the laboratory at Mahidol University in Bangkok, where individuals were identified to species level by use of the morphological keys of Buei ${ }^{18}$ and of Rattanarithikul and Panthusiri. ${ }^{19}$

Individual mosquitoes were blood-fed from hamsters and 
TABLE 1

Prevalence of Wolbachia infection in natural Aedes albopictus population sampling from Chachoengsao, eastern Thailand

\begin{tabular}{lcccc}
\hline \multirow{2}{*}{$\begin{array}{c}\text { Date of } \\
\text { collection }\end{array}$} & \multirow{2}{*}{$\begin{array}{c}\text { Total no. } \\
\text { with F1* }\end{array}$} & \multicolumn{3}{c}{ Infected, $n(\%) \dagger$} \\
\cline { 3 - 5 } & 180 & $145(100.00)$ & $0(0.00)$ & $0(0.00)$ \\
August 1999 & 180 & A only & B only \\
September 1999 & 255 & $232(99.15)$ & $2(0.85)$ & $0(0.00)$ \\
October 1999 & 267 & $259(99.23)$ & $2(0.77)$ & $0(0.00)$ \\
November 1999 & 142 & $137(98.56)$ & $2(1.44)$ & $0(0.00)$ \\
December 1999 & 161 & $161(100.00)$ & $0(0.00)$ & $0(0.00)$ \\
January 2000 & 76 & $76(100.00)$ & $0(0.00)$ & $0(0.00)$ \\
Total & 1,081 & $1,010(99.41)$ & $6(0.59)$ & $0(0.00)$ \\
\hline
\end{tabular}

* Total number of field females that laid eggs and screened via PCR for the presence of Wolbachia before excluding samples negative for the Wolbachia outer surface protein ( $w s p$ ) and the synaptic vesicle nuclear protein (SVNP) genes.

$\dagger$ Percentage infection of Wolbachia based on combined polymerase chain reaction (PCR) results of field females and their corresponding F1 progeny.

then allowed to lay eggs in a confined vial one-quarter filled with water. After egg laying, parent females were assayed by PCR for Wolbachia infection. Eggs from individual females were stored under optimal conditions in the insectary until the infection status of the female parent was determined. In the case that the female parent was determined to be infected with only one strain of Wolbachia or to be uninfected, the corresponding egg batch from this female was hatched by placing the eggs in deoxygenated water for 24 hr. The larvae were then transferred to rearing trays in the insectary and fed fish food until adult emergence. These F1 adults were PCR tested to confirm the infection status of their female parents.

Polymerase chain reaction typing of Wolbachia infection. We extracted DNA from the dissected ovaries of individual females. The extraction procedure followed the crude boiling method of O'Neill and others. ${ }^{20}$ Ovaries were dissected in sterile double-distilled water on a sterile microscope slide and were homogenized with $100 \mu \mathrm{L}$ of STE buffer $(100 \mathrm{mM} \mathrm{NaCl}, 10 \mathrm{mM}$ Tris- $\mathrm{HCl}, 1 \mathrm{mM}$ EDTA, pH 8.0). The homogenates were heated at $95^{\circ} \mathrm{C}$ for $10 \mathrm{~min}$ and centrifuged at $14,000 \mathrm{rpm}$ for $1 \mathrm{~min}$. One microliter of the supernatant was used in a $20-\mu \mathrm{L}$ PCR reaction. The DNA extraction from gonad tissues of the Wolbachia-infected colony of Ae. albopictus was used as a positive control. Negative PCR control, which consisted of PCR reactions without the addition of template, was included randomly to check for contamination.

The PCR amplification was carried out in $20-\mu \mathrm{L}$ reaction mixtures consisting of $2 \mu \mathrm{L} 25 \mathrm{mM} \mathrm{MgCl}, 2 \mu \mathrm{L} 10 \times$ buffer (Promega, Madison, WI), $0.5 \mu \mathrm{L} 20 \mu \mathrm{M}$ forward and reverse primers, $0.5 \mu \mathrm{L}$ dNTPs (10 mM each), 1 unit of Taq DNA polymerase (Promega), and $1 \mu \mathrm{L}$ of DNA template. The temperature profile started with an initial denaturation at $95^{\circ} \mathrm{C}$ for $3 \mathrm{~min}$, followed by $95^{\circ} \mathrm{C}$ for $1 \mathrm{~min}, 50^{\circ} \mathrm{C}$ for $1 \mathrm{~min}$, and $72^{\circ} \mathrm{C}$ for 1 min per cycle for 30 cycles. A PCR product of $10 \mu \mathrm{L}$ was electrophoresed with a 1-kb DNA ladder (Gibco BRL, Gaithersburg, MD) on a $1 \%$ agarose gel. The DNA bands were visualized by ethidium bromide staining.

Specific primers developed from the rapidly evolving wsp outer-surface protein gene of Wolbachia were used to screen for the presence of these bacteria in Ae. albopictus mosquitoes. ${ }^{21,22}$ The quality of DNA extraction was checked by use of primers for the synaptic vesicle nuclear protein gene
TABLE 2

Comparison of Wolbachia infection rate between field-collected female mosquitoes without F1 versus those with F1 confirmation

\begin{tabular}{llll}
\hline \multirow{2}{*}{$\begin{array}{c}\text { Infection } \\
\text { status }\end{array}$} & \multicolumn{2}{c}{$\begin{array}{c}\text { Infection rate in field-collected } \\
\text { female mosquitoes }\end{array}$} & \multicolumn{1}{c}{$\begin{array}{c}\text { Inaccuracy } \\
(\%)\end{array}$} \\
\cline { 2 - 3 } & \multicolumn{1}{c}{ Without F1 } & \multicolumn{1}{c}{ With F1 } & 1.48 \\
AB infected & $0.979(995 / 1,016)$ & $0.994(1,010 / 1,016)$ & 0.89 \\
A infected & $0.015(15 / 1,016)$ & $0.006(6 / 1,016)$ & 0.89 \\
B infected & $0.006(6 / 1,016)$ & $0.000(0 / 1,016)$ & 0.59 \\
\hline
\end{tabular}

(SVNP) (Ono M, O’Neill SL, unpublished data) (SVNP2F2: TGC GGT TTG TGG CGT ATT CTC AGT; SVNP2R2: CCT CCA CGG GTT CGA TTG TTT TG) to screen any samples that were negative with wsp gene primers. The SVNP primers amplify a single copy nuclear gene of the mosquito and provide a conservative control for DNA extraction quality. Any mosquitoes that were negative with both $w s p$ and SVNP primer sets were excluded from the data set. Mosquitoes that were negative with general wsp primers but positive with SVNP primers were scored as uninfected. Samples that were positive with general wsp primers were assigned into specific Wolbachia A and B groups by use of group-specific $w s p$ and $f t s Z$ primers. ${ }^{22,23}$

\section{RESULTS}

A total of 1,568 individuals of Ae. albopictus were collected from 3 adjacent locations in Hua Samrong Subdistrict, Plaeng Yao District, Chachoengsao Province, eastern Thailand. Out of 1,107 blood-fed females, 1,081 laid eggs and were screened via PCR to determine their Wolbachia infection status by use of general $w s p$ primers. Sixty-five individuals were negative for both $w s p$ and SVNP primers and were excluded from the data set. Table 1 shows the frequency of double and single infections of Wolbachia in the Ae. albopictus population sampled from Chachoengsao, eastern Thailand. Our PCR results showed that $100 \%$ of the mosquitoes sampled were infected with the A group Wolbachia strain. Prevalence of double infection was determined to be $99.41 \%$. Individuals carrying a single infection of group A Wolbachia were observed at a very low rate, ranging 0.77 $1.44 \%$ during the months September-November. Over the entire sampling period, the mean percentage of individuals carrying only the group A infection was $0.59 \%$. No individuals were found carrying a single infection of group B Wolbachia in this study. Similarly, no uninfected individuals were sampled.

According to the PCR results of female parents with no F1 confirmation, a total of $995(97.93 \%)$ of 1,016 samples were determined to be superinfected with both $\mathrm{A}$ and $\mathrm{B}$ strains of Wolbachia, whereas single A-infected and single B-infected individuals were estimated to be 1.48 and $0.59 \%$, respectively (Table 2). However, when the infection status of each female parent was confirmed by PCR testing of her F1 offspring, we found that the PCR result underestimated the superinfected individuals in the field by $1.48 \%$. A female that PCR tested as single infected was considered to be actually superinfected if any of her progeny were superinfected. All male and female F1 offspring of individual parent females that were previously screened as single B-infected 
were found to be superinfected with both A and B groups of Wolbachia. After combining the PCR data of both parents and F1 offspring, the more accurate superinfection prevalence in this natural population was estimated to be $99.41 \%$ $(1,010$ of 1,016$)$. In addition, only 6 single A-infected individuals were present in this population. No single B-infected individual or uninfected individual was detected, even though a large population of Ae. albopictus was sampled in this study $(n=1,016)$.

\section{DISCUSSION}

Our results show a Wolbachia infection frequency of $100 \%$ in natural populations of the mosquito vector Ae. albopictus. Of these mosquitoes, $99.41 \%$ were superinfected, and the remainder were infected with group A Wolbachia infection. This prevalence is higher than previous studies that have measured infection prevalence in Drosophila populations. ${ }^{15}$ It is possible that infection with 2 different strains of Wolbachia may contribute to the high fidelity of maternal transmission of Wolbachia in natural populations of this mosquito species. ${ }^{24}$

Many different species of mosquito vectors have been reported to be infected with different strains of Wolbachia. ${ }^{25}$ Therefore, in order to use Wolbachia to drive transmission blocking genes into these species, Wolbachia superinfections would be needed. ${ }^{13}$ The results of this study show that mosquito populations are capable of being superinfected with Wolbachia at extremely high frequency and suggest that segregation of single infected strains from superinfected mothers is not a common event.

In this study, no confirmed uninfected individuals were encountered despite extensive sampling. However, we did find 8 females that were negative with specific primers of both Wolbachia A and B groups. Unfortunately, all of these females died before laying eggs, so we could not check F1 progeny to confirm their infection status. However, these samples were not positive after PCR testing with the SVNP control primers and were finally excluded from our analysis. If they were truly negative, the rate of uninfected individuals would have been maximally estimated at $0.78 \%$ ( 8 of 1,024$)$, which is still extremely low.

As indicated by Turelli and Hoffmann, ${ }^{14}$ data on natural infection frequency is critical in order to evaluate the potential to use Wolbachia as a vehicle to modify insect vector populations. Our data provide evidence that Wolbachia is a better candidate to use for genetic control experiments of mosquitoes than previous studies focused on Drosophila suggest.

Acknowledgments: We thank Tanong Aimmak and Somboon Srimarat for their help in collecting field materials and Kitti Theinthong, Samnieng Theinthong, and Nutchaya Klinpikul for their insectary and laboratory assistance.

Financial support: This work was supported by the TRF/BIOTEC Special Program for Biodiversity Research and Training (BRT 139026) and the Thailand Research Fund (RTA/01/2541).

Authors' addresses: Pattamaporn Kittayapong and Visut Baimai, Department of Biology, Faculty of Science, Mahidol University, Rama 6 Road, Bangkok 10400, Thailand. Scott L. O’Neill, Section of Vector Biology, Department of Epidemiology and Public Health, Yale
University School of Medicine, 60 College Street, New Haven, CT 06520.

Reprint requests: Pattamaporn Kittayapong, Department of Biology, Faculty of Science, Mahidol University, Rama 6 Road, Bangkok 10400, Thailand, Telephone: 66-2-201-5254, Fax: 66-2-247-0079, E-mail: grpkt@mahidol.ac.th.

\section{REFERENCES}

1. Kambhampati S, Rai KS, 1991. Mitochondrial DNA variation within and among populations of the mosquito, Aedes albopictus. Genome 34: 288-292.

2. Kambhampati S, Black WC, Rai KS, 1991. Geographic origin of the US and Brazilian Aedes albopictus inferred from allozyme analysis. Heredity 67: 85-94.

3. Whitehead RH, Yuill TM, Gould DJ, Simasathien P, 1971. Experimental infection of Aedes aegypti and Aedes albopictus with dengue viruses. Trans $R$ Soc Trop Med Hyg 65: 661667.

4. Chan YC, Ho BC, Chan KL, 1971. Aedes aegypti (L.) and Aedes albopictus (Skuse) in Singapore City: V. Observations in relation to dengue haemorrhagic fever. Bull World Health Organ 44: 651-658.

5. Chow VT, Chan YC, Yong R, Lee KM, Lim LK, Chung YK, Lam-Phua SG, Tan BT, 1998. Monitoring of dengue viruses in field-caught Aedes aegypti and Aedes albopictus mosquitoes by a type-specific polymerase chain reaction and cycle sequencing. Am J Trop Med Hyg 58: 578-586.

6. Ibanez-Bernal S, Briseno B, Mutebi JP, Argot E, Rodriguez G, Martnez-Campos C, Paz R, de la Fuente-San Roman P, TapiaConyer R, Flisser A, 1997. First record in America of Aedes albopictus naturally infected with dengue virus during the 1995 outbreak at Reynosa, Mexico. Med Vet Entomol 11: 305-309.

7. Gould DL, Yuill TM, Moussa MA, Simasathien P, Rutledge LC, 1968. An insular outbreak of dengue haemorrhagic fever. III. Identification of vectors and observations on vector ecology. Am J Trop Med Hyg 17: 609-618.

8. Wright JD, Barr AR, 1980. The ultrastructure and symbiotic relationships of Wolbachia of mosquitoes of the Aedes scutellaris group. J Ultrastr Res 72: 52-64.

9. Kambhampati S, Rai KS, Verleye DM, 1992. Frequencies of mitochondrial DNA haplotypes in laboratory cage populations of the mosquito, Aedes albopictus. Genetics 132: 205209.

10. Kambhampati S, Rai KS, Burgun SJ, 1993. Unidirectional cytoplasmic incompatibility in the mosquito, Aedes albopictus. Evolution 47: 673-677.

11. Sinkins SP, Braig HR, O’Neill SL, 1995. Wolbachia superinfection and the expression of cytoplasmic incompatibility. Proc $R$ Soc Lond B 261: 325-330.

12. Sinkins SP, O'Neill SL, 2000. Wolbachia as a vehicle to modify insect populations. Handler AM, James AA, eds. Insect Transgenesis: Methods and Applications. Boca Raton, FL: CRC Press, 271-288.

13. Sinkins SP, Curtis CF, O'Neill SL, 1997. The potential application of inherited symbiont systems to pest control. O'Neill SL, Hoffmann AA, Werren JH, eds. Influential Passengers. New York: Oxford University Press, 155-175.

14. Turelli M, Hoffmann AA, 1999. Microbe-induced cytoplasmic incompatibility as a mechanism for introducing transgenes into arthropod populations. Insect Mol Biol 8: 243-255.

15. Turelli M, Hoffmann AA, 1995. Cytoplasmic incompatibility in Drosophila simulans: dynamics and parameter estimates from natural populations. Genetics 140: 1319-1338.

16. Jeyaprakash A, Hoy MA, 2000. Long PCR improves Wolbachia amplification: wsp sequences found in $76 \%$ of sixty-three arthropod species. Insect Mol Biol 9: 393-405.

17. Service MW, 1995. Mosquito Ecology: Field Sampling Methods. London: Chapman and Hall.

18. Buei K, 1983. Pictorial Key to Species, Adult Mosquitoes in Thailand. Bangkok: PPHS Project, Series No. 3, Ministry of Public Health. 
19. Rattanarithikul R, Panthusiri P, 1994. Illustrated Keys to the Medically Important Mosquitoes of Thailand. Bangkok: Wattana Panich Press.

20. O'Neill SL, Giordano R, Colbert AME, Karr TL, Robertson HM, 1992. 16S ribosomal RNA phylogenetic analysis of the bacterial endosymbionts associated with cytoplasmic incompatibility in insects. Proc Natl Acad Sci U S A 89: 26992702.

21. Braig HR, Zhou WG, Dobson SL, O’Neill SL, 1998. Cloning and characterization of a gene encoding the major surface protein of the bacterial endosymbiont Wolbachia pipientis. $J$ Bacteriol 180: 2373-2378.

22. Zhou W, Rousset F, O'Neill SL, 1998. Phylogeny and PCR clas- sification of Wolbachia strains using wsp gene sequences. Proc R Soc Lond B 265: 509-515.

23. Werren JH, Winsor D, Guo L, 1995. Distribution of Wolbachia among neotropical arthropods. Proc R Soc Lond B 262: 197204

24. Kittayapong P, Baisley KJ, Sharpe RG, Baimai V, O'Neill SL, 2001. Maternal transmission efficiency of Wolbachia superinfections in Aedes albopictus populations in Thailand. Am J Trop Med Hyg 66: 103-107.

25. Kittayapong P, Baisley KJ, Baimai V, O’Neill SL, 2000. Distribution and diversity of Wolbachia infections in Southeast Asian mosquitoes (Diptera: Culicidae). J Med Entomol 37: 340-345. 